Cahiers de recherches médiévales

Journal of medieval studies

3 | 1997

Espace vécu, mesuré, imaginé

\title{
Un espace révélé
}

Les miniatures du livre de Jean de Mandeville ms fr 2810

\section{Christine Bousquet-Labouérie}

\section{(2) OpenEdition}

Journals

Édition électronique

URL : https://journals.openedition.org/crm/2459

DOI : $10.4000 / \mathrm{crm} .2459$

ISSN : 1955-2424

Éditeur

Honoré Champion

Édition imprimée

Date de publication : 15 janvier 1997

Pagination : 71-78

ISSN : $1272-9752$

Référence électronique

Christine Bousquet-Labouérie, "Un espace révélé », Cahiers de recherches médiévales [En ligne], 31

1997, mis en ligne le 04 février 2008, consulté le 15 décembre 2022. URL : http://

journals.openedition.org/crm/2459; DOI : https://doi.org/10.4000/crm.2459 


\section{RM}

\section{Un espace révélé : les miniatures du livre de Jean de Mandeville ms fr 2810.}

John Mandeville's travel stages two most different spaces : one well known of biblic Neareast that the traveller has doubtlessly traversed, and the other one far Asia, very little known. The image-maker of the narration, through the staging of the discovery of the world, hands back the difference between these two areas. Ways and roads of a through-travelled East wear away, face to nowhere places, which the image-maker and the reader are confronted with. From a linear world, the illuminator leads to a circular world without beginning or end and reaches Mandeville's purpose to tell only one world.

Le manuscrit 2810 de la B.N., bien connu, voire parfois trop connu, est intéressant à bien des titres et en particulier au titre de notre sujet: espace vécu, mesuré, imaginé. Le récit central du manuscrit est celui du Voyage autour de la terre de Jean de Mandeville ${ }^{1}$. Le voyage du chevalier anglais met en scène deux espaces asiatiques complètement différents l'un de l'autre: le premier, la Terre Sainte, lieu connu par les Occidentaux, est raconté avec précision par le voyageur ; le second concerne l'Asie lointaine, celle du Khan et du Prêtre Jean. Le récit demeure plutôt évasif, l'auteur se contentant bien souvent de reprendre les informations contenues dans divers textes. en particulier dans celui d'Odéric de Pordenone $e^{2}$.

Le manuscrit 2810 contient le texte entier dans sa version insulaire ${ }^{3}$; ce manuscrit est orné de 74 grandes enluminures encadrées. La différence entre l'espace connu, voire même familier, du Proche-Orient biblique et celui, immense, d'une Asie parcourue par quelques clercs et quelques marchands partis à la suite des premiers explorateurs, transparaît dans le ton du récit. Il était alors intéressant de vérifier si l'imagier introduisait lui aussi une différenciation entre les espaces, entre les territoires décrits. L'étude de quelques unes des miniatures de notre manuscrit permet d'aborder ce qui se révèle être un espace vécu, mesuré et aussi imaginé. Comment un enlumineur rivé aux terres européennes a-t-il pu donner une mesure d'un espace qu'il imagine et dans lequel le voyageur est censé avoir vécu? Quel contenu concret donne-t-il à l'espace ou aux espaces différents arpentés ?

L'espace vécu est celui du voyageur réel ou supposé, c'est celui qui est marqué par des territoires facilement représentés, faciles à appréhender pour l'imagier qui, lui, est statique en Occident. Cet espace vécu s'inscrit donc dans un monde qu'il maîtrise de manière livresque, par exemple au travers des récits de pèlerinage pour la Terre Sainte. Il peut alors restituer cet espace de manière très

1 Jean de Mandeville, Voyage autour de la terre, éd. Ch.Deluz, les Belles lettres, Paris, 1993.

2 Cf Ch. Deluz, Le livre de Jehan de Mandeville, une géographie au XIV s., Louvain la Neuve, 1988 ; sur les sources de Mandeville, cf. annexe VI, pp. 429-431.

${ }^{3}$ J.Benett, The rediscovery of Sir John Mandeville. New York, 1954, appendix I, pp. 265334.

Cahiers de Recherches Médiévales (XIII'-XV s.). 3, 1997 
simple, en fonction de ses propres connaissances, dont la part de symbolique peut occuper une place non négligeable: dans ce cadre, on peut constater que certaine monuments et lieux sont reconnaissables: par exemple, la Montagne Sainte Catherine, gravie par Mandeville et son compagnon, est clairement identifiée et identifiable. La présence d'une église habritant la statue de sainte Catherine correspond à une appropriation de l'espace: par une forme connue, familière. L'imagier introduit le lecteur dans un espace maitrisé ou au moins reconnu (fig. 1$)^{4}$.

Le deuxième élément de cette image est la montagne proprement dite où " il n'y a ni église ni chapelle mais un monticule de pierres indiquant l'endroit où son corps fut déposé par les anges ${ }^{5}$. Le sarcophage ouvert et vide s'oppose à l'espace bâti connu et rassurant; il introduit le lecteur à l'espace imaginé qu'est celui de la mort. La différence entre ces deux mondes se marque par le chemin, bien net, porté par le bord du talus, et qui aboutit à l'église : les pèlerins l'ont emprunté. Puis, lors de l'ascension de la montagne, le chemin disparaît : la route passe à travers l'herbe sans trace d'une route concrètement dessinée. Le changement de dimension d'espace est accentuée par cette opposition entre un chemin tracé et une route à imaginer.

Le deuxième trait qui peut frapper le lecteur sur cette représentation est la forme donnée à la fois à l'image et à la route : bien que non marquée, elle conduit quelque part, sa structure est linéaire malgré les tours et détours imposés par la montagne. Cette montagne à gravir est un espace qui reste à explorer mais qui malgré tout est déjà presque domestiqué et mesurable.

Deux autres images du Livre de Mandeville restituent l'espace vécu, celui que l'on peut mesurer et celui qu'on ne peut plus mesurer: tout d'abord la grande miniature sur laquelle s'ouvre le récit (fig. 2$)^{\circ}$ : Mandeville prend congé du roi d'Angleterre, et, derrière son cheval bien campé sur un chemin qui ne mène nulle part, des rochers aiguisés et des arbres créent un nouvel espace : une route nouvelle débute et s'arrête tout de suite : l'ailleurs est indiqué par ces éléments naturels qui le balisent lorsque aucune route ni aucun chemin ne peuvent plus le restituer. Le monde mesuré à l'échelle de la ville et des murailles s'efface devant un monde démesuré où les unités ne sont plus les mêmes : le monde mesuré en milles devient celui arpenté en journées. Ce passage d'un espace aisément mesurable à celui, démesuré, de l'Asie a toujours été l'élément fondamental, premier de la découverte ; déjà près d'un siècle avant Jean de Mandeville. Guillaume de Rubrouck manifeste cet étonnement devant l'immensité des distances : ainsi du «lendemain de la fête de l'exaltation de la sainte Croix (le 15 septembre) nous commençâmes à chevaucher ; nous chevauchâmes continuellement vers l'Orient jusqu'à la fête de la Toussaint ${ }^{7}$. Et encore ne s'agissait-il que d'arriver au fleuve Oural! L'extrême Orient était encore loin derrière.

\footnotetext{
${ }^{4}$ Ms fr 2810 , fol. $153 \mathrm{v}^{\circ}$.

5 Mandeville, Voyage autour de la terre, éd. Ch. Deluz. p. 47.

- Ms fr 2810, fol. 141.

7 Guillaume de Rubrouck, Voyage dans l'empire mongol, éd. par Cl. et R. Kappler, Paris, Imprimerie Nationale, 1993, p. 219.
} 
Cette introduction à un autre monde est particulièrement claire sur la miniature du frontispice: la structure circulaire du premier plan et qui ouvre vers l'au-delà - derrière le voyageur - crée un nouvel espace à appréhender. En restituant ou en révélant un monde à explorer, l'image introduit le temps, temps du voyage, temps de l'image, dans lequel à la prise de congé succède immédiatement le temps de la découverte.

Dans le même esprit, nous pouvons regarder l'image de Mandeville cheminant vers Babylone (fig. 3$)^{8}$. Le récit précise que «cette cité de Babylone n'est pas la Grande Babylone celle où les divers langages furent confondus par un miracle de Dieu quand on avait commencé à faire la grande Tour de Babel ". Cette tour, continue le narrateur, «est abandonnée et il y a des dragons de grands serpents et diverses bêtes venimeuses tout autour ». Une fois encore, l'imagier restitue deux espaces : celui du chemin poursuivi par les trois voyageurs, qui apparait clairement dessiné et emprunté par les chevaux sortant de la ville. L'espace cadré, mesuré, vécu se détache clairement tandis que la tour - objet d'admiration de la part du voyageur qui la désigne à son compagnon d'un bras tendu - est clairement inaccessible, remparée qu'elle est par les montagnes. L'impossibilité d'accéder à l'espace imaginaire peuplé de dragons est mis en lumière par l'enceinte de rochers escarpés construisant un monde clos enserré entre les montagnes. Au centre de cette enceinte, se dresse la tour ronde et rose. La tour qui formait un carré d'après le récit, s'arrondit sous les mains de l'artiste accentuant ainsi ce monde clos imaginé qui ne correspond à aucune réalité. Mandeville ne dit-il pas quelques lignes plus loin que le roi Cyrus détruisit la cité et la tour? Le lien entre les deux espaces dans cette image est sans doute celui de ce chemin qui semble bien s'enfoncer entre les rochers mais qui n'est plus tracé, n'est plus apparent. La disparition du repère spatial le plus concret lie les deux mondes en les opposant fortement.

Ces trois premières images porteuses d'opposition, d'espaces différenciés, permettent à l'imagier de restituer à la fois des mondes vécus ou qu'il considère comme vécus par l'écrivain, et les mondes imaginés, ou du moins que lui ne peut qu'imaginer. Les trois images suivantes mettent en lumière, non plus des mondes en opposition, mais des espaces dans lesquels se déroulent soit des scènes invraisemblables pour l'Occidental, soit une quête d'un monde à définir.

La première scène illustre l'île de la Sicile. et l'Etna «qui brûle sans cesse $»^{10}$ (fig. 4) ; l'imagier, par ces flammes qui couronnent la montagne, illustre ces cheminées de l'enfer visibles depuis l'Italie. La présence, à l'arrière plan de l'image, du volcan jette le pont avec les côtes italiennes éloignées de vingt-cinq milles ; mais ce n'est pas là l'élément spatial essentiel. Le monde ouvert devant les yeux des lecteurs est un monde circulaire où il n'y a ni chemin ni route, ni ville: aucun élément concret rappelant un monde vécu. Ces hommes semblent être là de toute éternité. Ce monde enfermé dans les montagnes est un monde dangereux, étonnant

\footnotetext{
${ }^{8}$ Ms fr 2810, fol. 153.

${ }^{9}$ Mandeville, p. 30

${ }^{10}$ Mandeville, p. 42 et Ms fr 2810, fol. 155.
} 
et troublant pour l'Occidental qui désigne de la main la vision infernale lui apparaissant. Les hommes de Sicile, craignant la bâtardise de leurs enfants et cherchant de manière radicale la paternité de leurs rejetons présentent ceux-ci à des serpents.

S'ils sont nés de mariage régulier, le serpent les entoure sans leur faire aucun mal, mais s'ils sont bâtards, il les mord et leur instille son venin. Plusieurs hommes mariés cherchent ainsi si les enfants sont leurs"

L'imagier prend quelques libertés en introduisant des dragons ailés représentation conventionnelle des serpents, qui ne se contentent pas de mordre le pauvre malheureux bâtard, mais semblent bien le déchiqueter. Une telle vision ne parait pas faire grand effet sur le clan des Orientaux qui se contentent d'observer la scène sans prendre parti. L'espace de la scène est délimité par les ombres dans lesquelles surgissent les animaux monstrueux: le ciel très réduit, les rochers envahissants, les animaux et les ombres créent un lieu plein de mystère et de terreur accentué par le volcan menaçant, très noir, semblant barrer le monde, et qui s'oppose à un espace des adultes participants ou observateurs ; ce dernier espace, lumineux, mais lui aussi suspect, car surgi de nulle part.

Ces caractéristiques liées à un monde menaçant, incompatible avec l'humanité occidentale, ne sont pas liées uniquement au caractère monstrueux de la scène: on les retrouve aussi dans d'autres constructions d'espaces bénéfiques (fig. 5) ${ }^{12}$ : les deux hommes qui contemplent la Mer Aréneuse et la rivière rocheuse introduisent eux aussi un monde circulaire délimité par des rochers en deux plans successifs. Cette mer portant poisson alors qu'elle est sans eau, ce fleuve qui court trois fois par semaine en entraînant avec lui de grosses pierres disparaissant dès qu'elles sont entrées dans la Mer Arèneuse introduisent le lecteur dans un monde imaginé par l'artiste. L'absence de repères concrets, sensibles, permettant de fixer un espace, est ici aussi clairement apparente: le voyageur, au fond, semble sortir de nulle part. Les deux hommes au bord de l'image sont posés au bord du monde dont ils sont issus et qui, par cet unique tronc d'arbre coupé en plein centre de l'image barrière entre les deux mondes -, est celui de la vie, du par-deça et de l'au-delà. Ce rôle assigné à ce bout de bois est en relation avec la présence des deux diables accoudés sur la montagne sombre. Ces deux personnages inquiétants en plein centre de l'image, dominant le monde sur lequel ils s'accoudent, sont les maîtres des flots d'une rivière qui pourrait bien être infernale. L'absence de toute pierre précieuse dans les cailloux qui tracent un chemin perdu donne au cours de cette rivière un aspect sombre accentué par le ciel très réduit, la couleur très noire des rochers qui, comme dans l'image précédente, s'oppose à la lumière du monde de ceux qui qui n'ont pas franchi la ligne entre l'espace vécu et l'espace imaginé.

\footnotetext{
${ }^{11}$ Mandeville, p. 41.

${ }^{12}$ Ms fr 2810 , fol. 213
} 
Pour conclure sur ces quelques représentations de l'espace tel qu'un enlumineur le met en place, une image paraît porter l'idée-même d'une quête d'un monde à découvrir. Cette image, qui illustre bon nombre de livres et même de travaux universitaires, crée un espace circulaire, une fois encore, là où tous les repères ont pratiquement disparu. (fig. 6). Seuls deux rochers imposent une borne à un monde sans début ni fin, sans chemin, monde dans lequel ces bateaux semblent tourner indéfiniment. La construction de cette image unissant ciel et terre, où même les hommes disparaissent sur le navire le plus éloigné, donne naissance à un monde circulaire où connu et inconnu se succèdent. Les deux rochers, bornes du monde, se répondent et s'opposent ; l'un porteur de végétation et d'une faune, l'autre aride et désolé. On pourrait y voir la représentation de deux mondes qui s'opposent :celui de l'Orient, assimilé à la nature sauvage, et celui de l'Occident urbain, connu donc non représenté ; peut être, mais plus que deux espaces, les rochers ne seraient-ils pas les portes qui à la fois créent et limitent l'espace ? Celui-ci est pourtant maitrisé, et c'est là le seul élément de ce rapport de l'homme à l'espace qu'il appréhende : la boussole sur le bâteau au premier plan indique la domination de l'homme sur un monde encore inconnu pour beaucoup et qui généralement suscite la méfiance des Occidentaux. L'espace maritime très mal maitrisé est celui qui, de manière radicale, voit disparaître tous ces repères, celui qui crée une certaine angoisse et dans lequel il n'y a aucun chemin. Cette image illustre le chapitre primordial de Mandeville, celui où il donne la raison d'être de son livre: faire la preuve de la rotondité de la terre et de la mer à partir de l'île de Lamory :

En cette terre, comme en plusieurs autres de par delà, on ne voit pas l'étoile Tramontane, c'est l'étoile de mer, qui est vers le nord et ne bouge pas. Mais on en voit une autre, qui est à l'opposé de celle-ci, vers le midi et que l'on appelle Antarctique. Et de même que les mariniers d'ici prennent leur direction et se dirigent grâce à l'étoile vers le nord, de même les mariniers de là-bas le font grâce à cette étoile vers le midi, qui ne nous apparaît pas et celle vers le nord ne leur apparaît pas. C'est pour cela que l'on peut s'apercevoir que la terre et la mer sont de forme ronde, car la partie du firmament qui apparait dans un pays n'apparaît pas dans l'autre. Et on peut bien prouver par l'expérience et par de subtiles indications que, si l'on pouvait s'embarquer sur un navire et trouver des gens qui veuillent aller à la découverte du monde. l'on pourrait aller sur ce navire tout autour du monde, et dessus, et dessous ${ }^{13}$.

Et Mandeville d'étayer son propos par des mesures qu'il dit avoir fait luimême, astrolabe en main ${ }^{14}$, avec cet instrument qui serait peut-être une boussole ${ }^{15}$. Etoiles, astrolabe et boussole lancent l'invitation à aller plus loin, à la découverte à la

\footnotetext{
${ }^{13}$ Mandeville, p 137.

${ }^{14}$ Ibid., p. 138.

${ }^{15} \mathrm{G}$.Beaujouan et E. Poulle, "Les origines de la navigation astronomique aux $\mathrm{XIV}$ et $\mathrm{XV}^{\mathrm{e}}$ siècles", Le Navire et l'économie maritime du XVème au XVIIème siècles, Travaux du colloque d'histoire maritime du 17 mai 1956. p. 103. planche XII, pp. 112-113. Les auteurs contredisent la légende traditionnelle d'une navigation à l'astrolabe. Il s'agit bien d'une navigation à la boussole.
} 
suite du récit. Mais le bateau qui s'enfuit au fond, vide de tout occupant, symbolise la peur des hommes à l'idée d'entreprendre un tel voyage.

La progression des images dans le récit imagé du livre de Jehan de Mandeville fait passer le lecteur d'un monde dessiné, portraituré pratiquement où les villes sont remparées, où les églises sont gothiques, où les chemins ressemblent à des routes, à un monde circulaire borné et suspect pour aboutir à la construction d'un monde rond où l'image rejoint le but essentiel de Mandeville : dire un seul monde.

Christine Bousquet-Labouérie

(Université de Tours)

\section{****** \\ ILLUSTRATIONS}

fig. 1 : BNF, $m s$ fr 2810 , fol. $153 v^{\circ}$

fig. 2 : BNF, $m s$ fr 2810 , fol. 141

fig. 3 : BNF, $m$ s fr 2810 , fol. 153

fig. 4 : BNF. ms fr 2810 , fol. 155

fig. 5 : BNF, $m$ fr 2810 . fol. 213

fig. 6 : BNF, $m s$ fr 2810 , fol. 188 


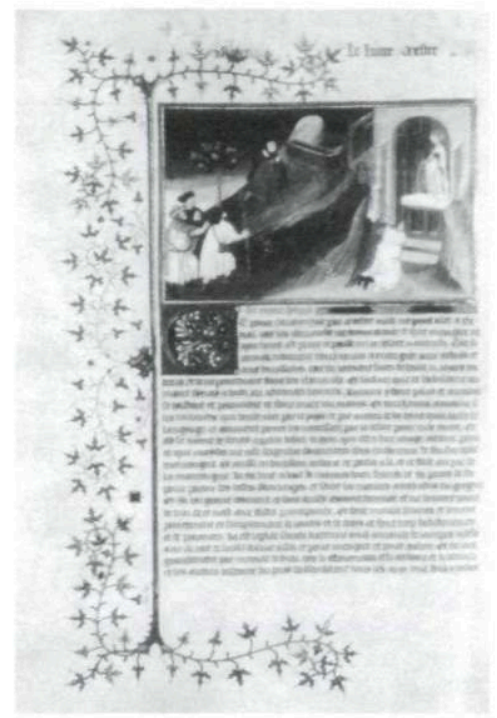

fig. 1

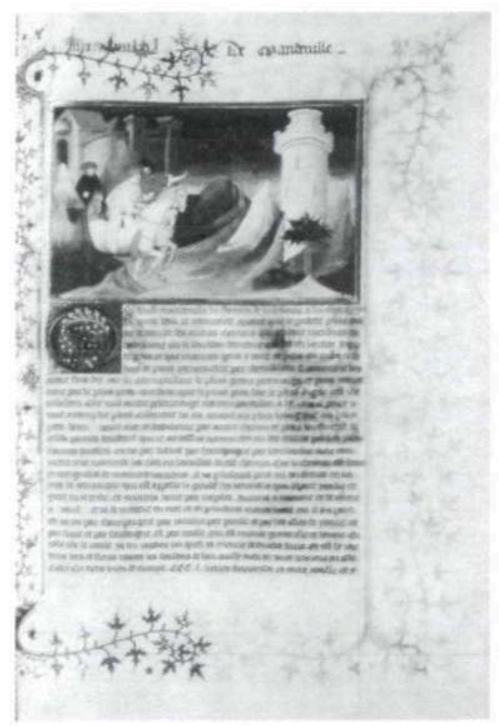

fig. 2

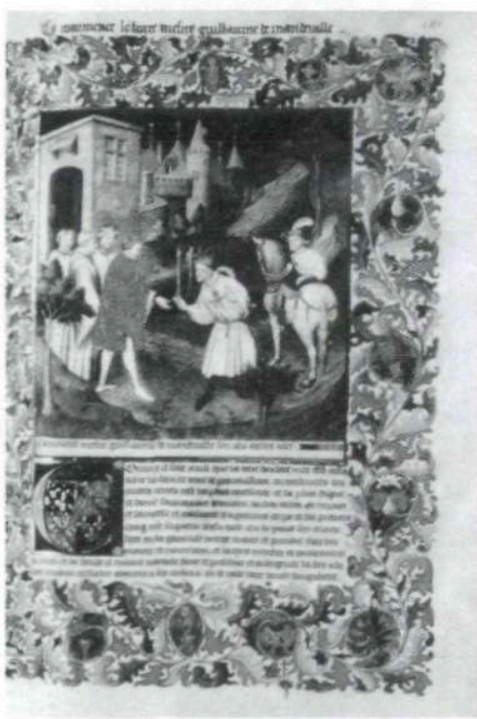

fig. 3 


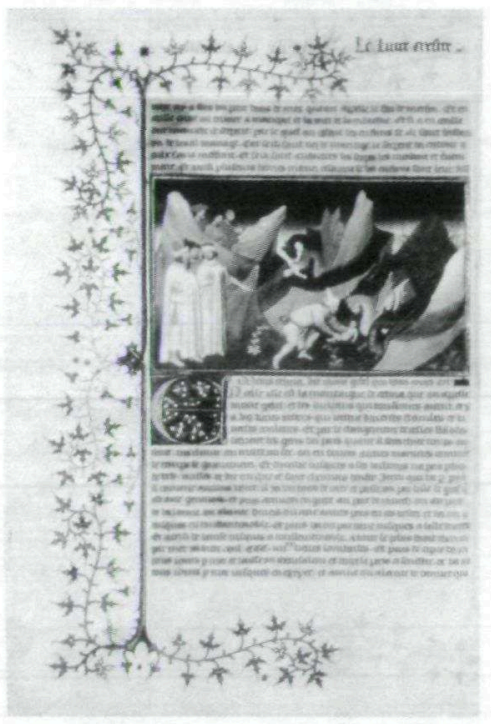

fig. 4

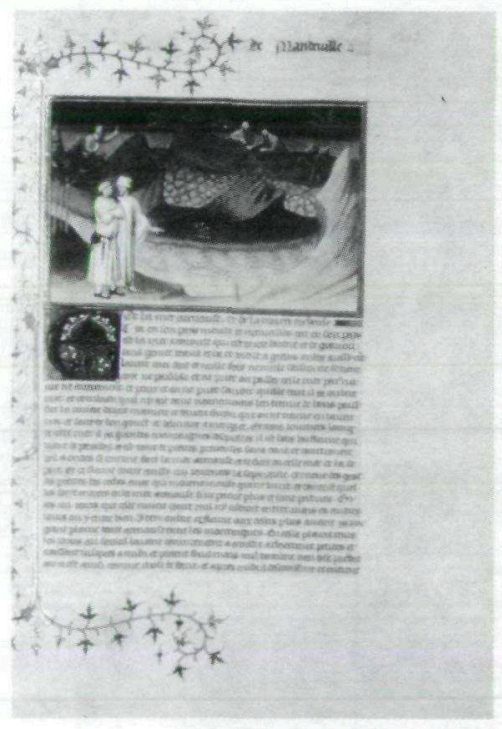

fig. 5

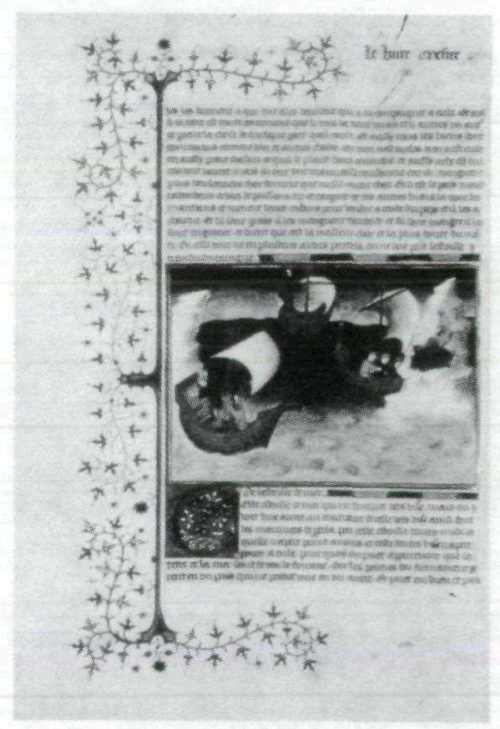

fig. 6 